\title{
Gemfibrozil attenuates the inflammatory response and protects rats from abdominal sepsis
}

\author{
CARLOS R. CÁMARA-LEMARROY ${ }^{1}$, FRANCISCO J. GUZMAN-DE LA GARZA ${ }^{2}$, PAULA CORDERO-PEREZ ${ }^{3}$, \\ JUAN M. IBARRA-HERNANDEZ ${ }^{2}$, LINDA E. MUÑOZ-ESPINOSA ${ }^{3}$ and NANCY E. FERNANDEZ-GARZA ${ }^{2}$ \\ ${ }^{1}$ Department of Internal Medicine, University Hospital 'José Eleuterio González', Autonomous University of Nuevo León; \\ ${ }^{2}$ Department of Physiology, School of Medicine, Autonomous University of Nuevo León; \\ ${ }^{3}$ Liver Unit, Department of Internal Medicine, University Hospital 'José Eleuterio González', \\ Autonomous University of Nuevo León, Monterrey, Nuevo León 64460, Mexico
}

Received May 6, 2014; Accepted November 28, 2014

DOI: $10.3892 /$ etm.2015.2190

\begin{abstract}
Sepsis is a serious condition characterized by an infectious process that induces a severe systemic inflammatory response. In this study, the effects of gemfibrozil (GFZ) on the inflammatory response associated with abdominal sepsis were investigated using a rat model of cecal-ligation and puncture (CLP). Male Wistar rats were randomly divided into three groups: Sham-operated group (sham), where laparotomy was performed, the intestines were manipulated, and the cecum was ligated but not punctured; control group, subjected to CLP; and GFZ group, which received GFZ prior to undergoing CLP. The groups were then subdivided into three different time-points: 2, 4 and $24 \mathrm{~h}$, indicating the time at which blood samples were obtained for analysis. Serum concentrations of tumor necrosis factor- $\alpha$ (TNF- $\alpha)$, interleukin-1 (IL-1), malondialdehyde (MDA), aspartate aminotransferase (AST), alanine aminotransferase (ALT) and lactate dehydrogenase (LDH) were determined. The LDH, AST and ALT values were significantly elevated following CLP compared with those in the sham group, and GFZ treatment was able to reduce these elevations. GFZ also reduced the sepsis-induced elevations of TNF- $\alpha$ and IL-1. In conclusion, GFZ treatment was able to attenuate the inflammatory response associated with CLP-induced sepsis, by diminishing the release of inflammatory cytokines, thereby reducing tissue injury and oxidative stress.
\end{abstract}

Correspondence to: Dr Carlos R. Cámara-Lemarroy, Department of Internal Medicine, University Hospital 'José Eleuterio González', Autonomous University of Nuevo León (UANL), Av. Francisco I. Madero y Dr. Eduardo Aguirre Pequeño S/No, Col. Mitras Centro, Monterrey, Nuevo León 64460, Mexico

E-mail: crcamara83@hotmail.com

Key words: sepsis, gemfibrozil, fibrates, inflammation, rat

\section{Introduction}

Sepsis is a serious condition associated with great mortality, characterized by an infectious process that induces a severe systemic inflammatory response. The physiopathology of sepsis is well understood, and mediators such as pro-inflammatory cytokines, reactive oxygen species, nitric oxide, toll-like receptors and transcription factors such as nuclear factor $\kappa \mathrm{B}$ (NF- $\mathrm{kB}$ ) play central roles in regulating the immune response responsible for tissue injury and circulatory collapse $(1,2)$. These events lead to hypotension, increased capillary permeability, multiple organ failure and mortality.

Although various therapeutic strategies have been implemented with the aim of reducing sepsis-associated morbidity and mortality, the cornerstone of treatment remains prompt antibiotic use and hemodynamic management (3). The use of immunomodulators, such as activated protein $\mathrm{C}$, has been evaluated and early results were promising (4). However, a recent trial did not find any clinical benefit with the use of this drug (5), thus questioning the use of drugs that attempt to modulate the inflammatory response. Various drugs with pleiotropic immunomodulatory properties are currently being studied, and this remains an active field of research (6).

Fibrates such as gemfibrozil (GFZ) and fenofibrate are drugs commonly used in the management of dyslipidemia. They are ligands for peroxisome proliferator-activated receptor $\alpha$ (PPAR- $\alpha$ ), receptors that belong to the steroid nuclear receptor family and that participate in lipid peroxidation, the cell cycle and fatty acid synthesis. Potent immunomodulatory effects have been associated with fibrates (7). Fibrates have shown the ability to reduce the expression of inflammatory genes in endothelial cells, the release of pro-inflammatory cytokine such as interleukins (ILs) and tumor-necrosis factor- $\alpha$ (TNF- $\alpha$ ), and the transcription of NF- $\mathrm{KB}$ in experimental studies $(8,9)$. Fibrates have also been shown to reduce IL-1 and C-reactive protein in humans (10). Fibrates also possess antioxidant effects, and regulate the activation and function of inflammatory cells $(11,12)$. These effects are independent of the lipid-lowering properties of fibrates.

The anti-inflammatory effects of fibrates have been tested in various experimental models of tissue and organ 
injury, showing beneficial results (reduced injury and inflammation) in models of autoimmune encephalomyelitis (13), ischemia-reperfusion injury (14) and alcohol-induced hepatotoxicity (15), among others. Considering the wide ranging immunomodulatory effects of fibrates, some of which overlap with the physiopathology of sepsis, the use of fibrates emerges as an interesting option.

\section{Materials and methods}

Animals. Animal procedures were performed in accordance with the proper use and care of laboratory animals, approved by the ethics committee of the University Hospital 'José Eleuterio González' (Monterrey, Mexico). Experiments were performed on 45 male Wistar rats weighing 200-250 g (Vivarium of the Department of Physiology, Autonomous University of Nuevo León, Monterrey, Mexico). Animals were maintained under standard conditions, including a stable room temperature $\left(24 \pm 3^{\circ} \mathrm{C}\right)$, a $12 \mathrm{~h}$ light/12 h dark cycle, and had access to commercial rat pellets and water ad libitum.

Cecal ligation and puncture model (CLP) (16). After fasting for $12 \mathrm{~h}$, the rats underwent ketamine/xylazine anesthesia (Anesket; Pfizer Inc., Mexico City, Mexico) at a dose of $50 / 10 \mathrm{mg} / \mathrm{kg}$, intraperitoneally (i.p). Animals were placed under a heating lamp in order to preserve a core body temperature of $37^{\circ} \mathrm{C}$. A midline incision exposed the intestines and the cecum was ligated (using 2-0 silk sutures) immediately proximal to the ileocecal valve causing a $50 \%$ obstruction and allowing for permeability. Two through-and-through punctures using a 18-gauge needle were performed at the anti-mesenteric side of the cecum. Light pressure was applied to confirm that fecal matter could emerge into the peritoneal cavity. The cecum was returned to its site, the wound was closed and $3 \mathrm{ml} / 100 \mathrm{~g}$ saline was administered subcutaneously.

The rats were randomly divided into three groups $(n=15$ per group): i) Sham-operated group (sham), where laparotomy was performed, the intestines were only manipulated and the cecum was ligated but not punctured. ii) Control group, subjected to CLP as described above. iii) GFZ group that received GFZ (Pfizer Inc.) prior to undergoing CLP. A dose of $100 \mathrm{mg} / \mathrm{kg}$ GFZ was chosen based on dose-response experiments and was administered $24 \mathrm{~h}$ and immediately prior to surgery. This dose has been shown to decrease oxidative stress in rodent models (11). The groups were then subdivided into three different time-points: 2, 4 and $24 \mathrm{~h}$. These time-points indicated the time after CLP when samples were obtained for analysis.

Serum analysis. Blood samples obtained following CLP were used to determine the serum levels of aspartate aminotransferase (AST), alanine aminotransferase (ALT) and lactate dehydrogenase (LDH) by standard biochemical automated methods (Vitros Chemical Products; Johnson \& Johnson, New Brunswick, NJ, USA), using commercially available kits and DT6011 and DTSC11 analyzers (Vitros Chemical System; Johnson \& Johnson). The serum concentrations of TNF- $\alpha$ and IL-1 were determined using a rat ELISA kit (PeproTech, Mexico City, Mexico). Lipid peroxidation, expressed as the malondialdehyde (MDA) level, was assessed by the thiobar- bituric acid reactive substances (TBARS) method using a TBARS colorimetric assay kit (Cayman Chemical Company, Ann Arbor, MI, USA). The level of LDH was only evaluated at the 24-h time-point after CLP.

Survival. To assess survival, additional groups of rats ( $n=10$ per group) underwent the same study protocol as those in the control and GFZ groups, and the rats were left to recover following the surgery. The animals were observed for a period of five days and the mortality rate was recorded.

Statistical analysis. SPSS statistical software, version 11.0 (SPSS Inc., Chicago, IL, USA) was used to analyze data using one-way analysis of variance (ANOVA) and with LSD post-hoc test (when data were found to be normally distributed) and Kruskal-Wallis test (when data were not normally distributed) so as to evaluate comparisons between groups, and differences between groups, respectively. The survival curves were determined using the Kaplan-Meier method and the log-rank test was used to compare the curves. All values are expressed as mean \pm standard deviation (SD) and $\mathrm{P}<0.05$ was considered to indicate a statistically significant result.

\section{Results}

ALT and AST. AST and ALT values were significantly elevated following CLP compared with those in the sham group, peaking at $4 \mathrm{~h}$ for AST and $24 \mathrm{~h}$ for ALT (Table I). GFZ treatment was able to reduce the elevations in AST and ALT, with its effects reaching statistical significance at 2 and $4 \mathrm{~h}$ in the case of AST and at $4 \mathrm{~h}$ in the case of ALT. At $24 \mathrm{~h}$ after CLP, LDH levels were significantly elevated, and GFZ treatment was also able to attenuate this increase.

Serum cytokine levels. Levels of TNF- $\alpha$ were significantly elevated in all time-points in the CLP group as compared with those in the sham controls (Fig. 1). TNF- $\alpha$ levels peaked at $4 \mathrm{~h}$ and remained elevated even at $24 \mathrm{~h}$. GFZ treatment resulted in significantly lower levels of TNF- $\alpha$ at 4 and $24 \mathrm{~h}$ after CLP. In the case of IL-1, there was no difference between the groups at $2 \mathrm{~h}$, where levels were undetectable by the assay. At 4 and $24 \mathrm{~h}$, the IL-1 levels were elevated; however, statistical significance was reached only at $24 \mathrm{~h}$. At this time-point, GFZ reduced IL-1 levels significantly (Fig. 1).

$M D A$. There were two identifiable time-points of increased MDA levels in the CLP group, at 2 and $24 \mathrm{~h}$. However, no difference was found between the CLP and GFZ groups (data not shown).

Survival. By $48 \mathrm{~h}$, five rats in the CLP group and three in the GFZ group had died. At 5 days, seven rats in the CLP group and five rats in the GFZ group had died. The difference in survival between groups did not reach statistical significance $(\mathrm{P}=0.1)$.

\section{Discussion}

PPARs have been studied in models of sepsis, and PPAR- $\gamma$ agonists have been found to be effective in reducing the 
Table I. Serum levels of ALT, AST and LDH.

\begin{tabular}{llll}
\hline Group & AST (UI) & ALT (UI) & LDH (UI) \\
\hline Sham & $109.0 \pm 10.6$ & $15.8 \pm 5.2$ & $537.0 \pm 141.4$ \\
CLP 2 h & $271.4 \pm 58.7^{\mathrm{a}}$ & $27.4 \pm 10.2$ & $39.8 \pm 16.3$ \\
CLP 4 h & $293.8 \pm 113.9^{\mathrm{a}}$ & $44.0 \pm 2.7^{\mathrm{a}}$ & $1983.4 \pm 530.7^{\mathrm{a}}$ \\
CLP 24 h & $200.8 \pm 16.6^{\mathrm{a}}$ & $19.0 \pm 11.6$ & $19.4 \pm 5.1^{\mathrm{b}}$ \\
GFZ 2 h & $175.8 \pm 69.9^{\mathrm{b}}$ & $37.4 \pm 8.3$ & $1057.4 \pm 403.2^{\mathrm{b}}$ \\
GFZ 4 h & $204.6 \pm 28.8^{\mathrm{b}}$ & $172.2 \pm 33.9$ &
\end{tabular}

Values are expressed as mean \pm standard deviation. ALT, alanine aminotransferase; AST, aspartate aminotransferase; LDH, lactate dehydrogenase; ${ }^{\mathrm{P}}<0.05$ vs. Sham; ${ }^{\mathrm{P}}<<0.05$ vs. CLP. GFZ, gemfibrozil; CLP, cecal ligation and puncture.
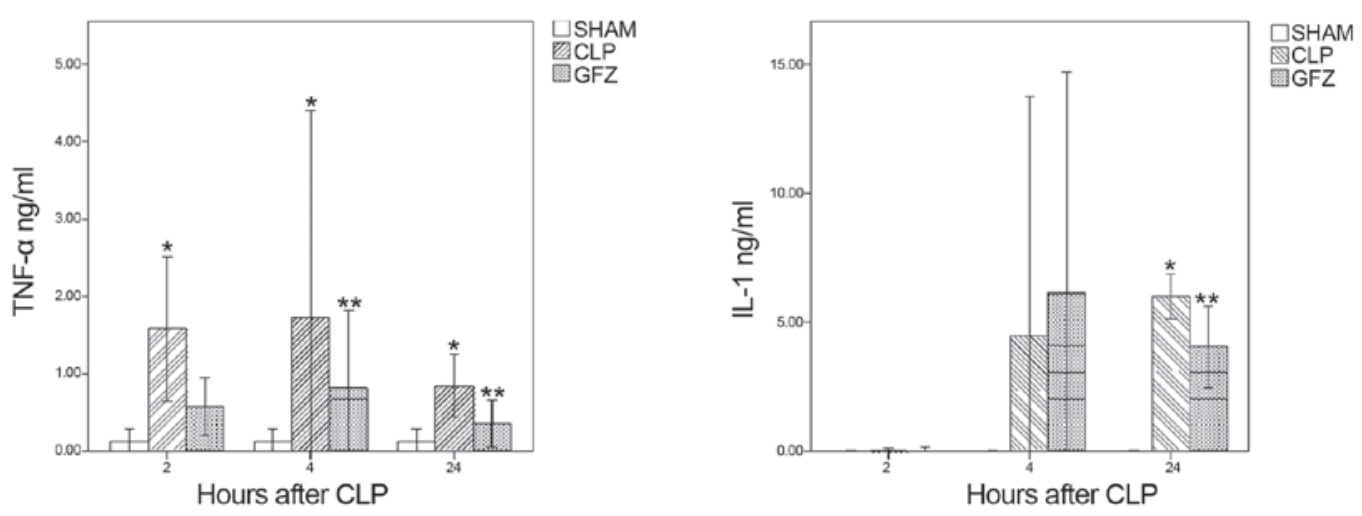

Figure 1. Levels of inflammatory cytokines. "P<0.05 vs. SHAM; ** P<0.05 vs. CLP. TNF, tumor necrosis factor; IL-1, interleukin-1; GFZ, gemfibrozil; CLP, cecal ligation and puncture.

severity of endotoxic shock (17). A recent study showed that PPAR- $\alpha$ expression was decreased in patients with septic shock and that the values correlated with the severity of disease; in addition, in the CLP model, knockout mice lacking PPAR- $\alpha$ had decreased survival rates compared with wild-type animals (18). Fenofibrate treatment has been shown to reduce endothelial dysfunction and endothelial cell injury in a rabbit model of endotoxemia by lipopolysaccharide (LPS) injection (19). Improved endothelial-dependent relaxation and decreased monocyte tissue factor expression were observed. Another model of LPS-induced endotoxemia in rats also demonstrated reductions in myocardial contractility depression and in TNF- $\alpha$ levels after fenofibrate treatment (20). These results are in contrast to those in another study where dietary fenofibrate caused increased levels of TNF- $\alpha$ and mortality in endotoxemic wild-type mice, while showing lower TNF- $\alpha$ levels in PPAR- $\alpha$ knockout mice (21). Using a physiologically relevant model of abdominal sepsis, in the present study it was demonstrated that GFZ pretreatment reduced the inflammatory response associated with abdominal sepsis. GFZ had immunomodulatory effects, reducing the circulating levels of TNF- $\alpha$, IL-1 and MDA, as well as markers of tissue injury such as AST and ALT. Differences between the LPS injection and CLP models could be relevant in explaining these inconsistent results, although the present study would support those showing a beneficial effect of PPAR- $\alpha$ ligands such as fibrates, despite only a trend towards a survival benefit being observed.

Inflammatory cytokines play an important but complex role in sepsis $(1,2,22)$. Some of the mechanisms by which fibrates might suppress inflammatory cytokines are beginning to be uncovered. In a study with LPS-activated cells, PPAR- $\alpha$ ligands, including fibrates, were able to inhibit NF- $\kappa$ B DNA binding activity in astrocytes, leading to a modulation of the expression of inflammatory genes responsible for the production of cytokines such as IL-1 and TNF- $\alpha$ (23). Similar results were obtained in LPS-stimulated cardiac myocytes (24), and in injured endothelial cells (9). Clinical studies have shown that GFZ reduces TNF- $\alpha$ production in human peripheral blood mononuclear cells (25), and fibrates reduce IL-1 in human whole blood stimulated by endotoxin (26). Fibrates dose-dependently inhibit cytokine production (ILs, TNF- $\alpha$ and interferons) in activated $\mathrm{T}$ cells, probably via the inhibition of transcription factors associated with the inflammatory response, such as activator protein-1, c-Jun NH2-terminal protein kinase and P38 mitogen-activated protein kinase (27). The expression of IL-1 receptor antagonist, which is acutely stimulated by LPS treatment in the liver, can also be induced by PPAR $\alpha$ (28). The switching of T cells from Th1 to Th2 profiles by GFZ is responsible for the tissue protection afforded against experimental encephalomyelitis in rats (12). Interestingly, the majority of studies use LPS, a key mediator 
of the sepsis inflammatory cascade, as a stimulant for cytokine production. In one study, using LPS-stimulated airway inflammation, PPAR- $\alpha^{-/-}$mice exhibited increased neutrophil infiltration and TNF- $\alpha$ production compared with that in PPAR $-\alpha^{+/+}$mice, and fenofibrate was able to reduce TNF- $\alpha$ production in wild-type mice (8).

$\mathrm{NF}-\kappa \mathrm{B}$ is a key mediator of the sepsis inflammatory cascade. NF- $\kappa \mathrm{B}$ activity is markedly increased in every organ studied, both in human and experimental models of septic shock, and greater levels of NF- $\kappa \mathrm{B}$ activity are associated with a higher rate of mortality and worse clinical outcome in septic patients (29). The inhibition of NF- $\kappa \mathrm{B}$, and of the subsequent overproduction of inflammatory cytokines such as TNF- $\alpha$, appears to be a crucial step in the immunomodulatory effects of PPAR- $\alpha$ activators. Cultured injured endothelial cells show activation of NF- $\mathrm{NB}$ that can be inhibited by incubation with fenofibrate (9). Fibrates were able to induce the expression of the inhibitory protein $\mathrm{I} \kappa \mathrm{B} \alpha$ in human aortic smooth muscle cells as well as in primary human hepatocytes, providing a possible mechanism for NF- $\mathrm{BB}$ inhibition (30). Another study has also linked TLR to PPAR- $\alpha$ signaling. The PPAR $\alpha$ agonist fenofibrate was found to reduce inflammatory cytokines and inflammation by antagonizing LPS-mediated inflammatory responses in vascular smooth muscle cells through a mechanism involving TLR-4 (31).

Marked oxidative stress results from the initiation of the inflammatory response in sepsis, and it initiates changes in mitochondrial function that may result in organ damage (32). Sepsis suppresses free fatty acid oxidation with the result of increased circulating fatty acids, through LPS-induced suppression of PPAR- $\alpha$ (33). Additionally, LPS induces the production of MDA and depletes catalase and superoxide dismutase in inflammatory cells (34). A previous study found elevations in MDA and glutathione depletion following CLP in rats (35). Fibrates are known to diminish MDA production in diabetic rats (36), and following severe liver ischemia-reperfusion injury in rats, fibrate pretreatment is able to reduce elevations in MDA and depletion of endogenous antioxidants (37). Elevations in MDA after CLP were observed in the present study, but only in untreated animals. No difference in MDA levels was identified between the CLP and GFZ group.

Although the inhibition of single cytokines or the addition of antioxidants has not shown value in the treatment of sepsis $(38,39)$, the use of immunomodulators with pleiotropic effects has been continuously studied (6). Lipid-lowering drugs such as statins are currently thought to be prime candidates as adjunct treatments in sepsis (40), and clinical trials are underway. In this study it was found that another class of lipid-lowering drugs, fibrates, also have immunomodulating properties and could also be of value. The use of these drugs, alone or in combination, warrants further study.

\section{Acknowledgements}

The authors would like to thank the staff at their laboratories for invaluable assistance.

\section{References}

1. Nduka OO and Parrillo JE: The pathophysiology of septic shock. Crit Care Clin 25: 677-702, 2009.
2. Cinel I and Opal SM: Molecular biology of inflammation and sepsis: a primer. Crit Care Med 37: 291-304, 2009.

3. Dellinger RP, Levy MM, Carlet JM, et al: Surviving sepsis campaign: international guidelines for management of severe sepsis and septic shock: 2008. Intensive Care Med 34: 17-60, 2008.

4. Neyrinck AP, Liu KD, Howard JP and Matthay MA: Protective mechanisms of activated protein $\mathrm{C}$ in severe inflammatory disorders. Br J Pharmacol 158: 1034-1047, 2009.

5. Ranieri VM, Thompson BT, Barie PS, et al; PROWESS-SHOCK Study Group: Drotrecogin alfa (activated) in adults with septic shock. N Engl J Med 366: 2055-2064, 2012.

6. Parrish WR, Gallowitsch-Puerta M, Czura CJ and Tracey KJ: Experimental therapeutic strategies for severe sepsis: mediators and mechanisms. Ann NY Acad Sci 1144: 210-236, 2008.

7. Michalik L and Wahli W: Involvement of PPAR nuclear receptors in tissue injury and wound repair. J Clin Invest 116: 598-606, 2006.

8. Delayre-Orthez C, Becker J, Guenon I, Lagente V, Auwerx J, Frossard N and Pons F: PPARalpha downregulates airway inflammation induced by lipopolysaccharide in the mouse. Respir Res 6: 91, 2005.

9. Yang TL, Chen MF, Luo BL, Xie QY, Jiang JL and Li YJ: Fenofibrate decreases asymmetric dimethylarginine level in cultured endothelial cells by inhibiting NF-kappaB activity. Naunyn Schmiedebergs Arch Pharmacol 371: 401-407, 2005.

10. Zambon A, Gervois P, Pauletto P, Fruchart JC and Staels B: Modulation of hepatic inflammatory risk markers of cardiovascular diseases by PPAR-alpha activators: clinical and experimental evidence. Arterioscler Thromb Vasc Biol 26: 977-986, 2006

11. Ozansoy G, Akin B, Aktan F and Karasu C: Short-term gemfibrozil treatment reverses lipid profile and peroxidation but does not alter blood glucose and tissue antioxidant enzymes in chronically diabetic rats. Mol Cell Biochem 216: 59-63, 2001

12. Roy A and Pahan K: Gemfibrozil, stretching arms beyond lipid lowering. Immunopharmacol Immunotoxicol 31: 339-351, 2009.

13. Dasgupta S, Roy A, Jana M, Hartley DM and Pahan K: Gemfibrozil ameliorates relapsing-remitting experimental autoimmune encephalomyelitis independent of peroxisome proliferator-activated receptor-alpha. Mol Pharmacol 72: 934-946, 2007.

14. Sivarajah A, Chatterjee PK, Hattori Y, et al: Agonists of peroxisome-proliferator activated receptor-alpha (clofibrate and WY14643) reduce renal ischemia/reperfusion injury in the rat. Med Sci Monit 8: BR532-BR539, 2002.

15. Nanji AA, Dannenberg AJ, Jokelainen K and Bass NM: Alcoholic liver injury in the rat is associated with reduced expression of peroxisome proliferator-alpha (PPARalpha)-regulated genes and is ameliorated by PPARalpha activation. J Pharmacol Exp Ther 310: 417-424, 2004.

16. Hubbard WJ, Choudhry M, Schwacha MG, Kerby JD, Rue LW III, Bland KI and Chaudry IH: Cecal ligation and puncture. Shock 24 (Suppl 1): 52-57, 2005.

17. Wu WT, Lee CC, Lee CJ, Subeq YM, Lee RP and Hsu BG: Rosiglitazone ameliorates endotoxin-induced organ damage in conscious rats. Biol Res Nurs 13: 38-43, 2011.

18. Standage SW, Caldwell CC,Zingarelli B and Wong HR: Reduced peroxisome proliferator-activated receptor $\alpha$ expression is associated with decreased survival and increased tissue bacterial load in sepsis. Shock 37: 164-169, 2012

19. Wiel E, Lebuffe G, Robin E, et al: Pretreatment with peroxysome proliferator-activated receptor alpha agonist fenofibrate protects endothelium in rabbit Escherichia coli endotoxin-induced shock. Intensive Care Med 31: 1269-1279, 2005.

20. Jozefowicz E, Brisson H, Rozenberg S, et al: Activation of peroxisome proliferator-activated receptor-alpha by fenofibrate prevents myocardial dysfunction during endotoxemia in rats. Crit Care Med 35: 856-863, 2007.

21. Hill MR, Clarke S, Rodgers K, Thornhill B, Peters JM, Gonzalez FJ and Gimble JM: Effect of peroxisome proliferator-activated receptor alpha activators on tumor necrosis factor expression in mice during endotoxemia. Infect Immun 67: 3488-3493, 1999.

22. van der Poll T and van Deventer SJ: Cytokines and anticytokines in the pathogenesis of sepsis. Infect Dis Clin North Am 13: 413-426, 1999.

23. Xu J, Chavis JA, Racke MK and Drew PD: Peroxisome proliferator-activated receptor-alpha and retinoid $\mathrm{X}$ receptor agonists inhibit inflammatory responses of astrocytes. J Neuroimmunol 176: 95-105, 2006 
24. Takano H, Nagai T, Asakawa M, et al: Peroxisome proliferator-activated receptor activators inhibit lipopolysaccharide-induced tumor necrosis factor-alpha expression in neonatal rat cardiac myocytes. Circ Res 87: 596-602, 2000.

25. Rosenson RS: Effect of fenofibrate on adiponectin and inflammatory biomarkers in metabolic syndrome patients. Obesity (Silver Spring) 17: 504-509, 2009.

26. Zhao SP, Ye HJ, Zhou HN, Nie S and Li QZ: Gemfibrozil reduces release of tumor necrosis factor-alpha in peripheral blood mononuclear cells from healthy subjects and patients with coronary heart disease. Clin Chim Acta 332: 61-67, 2003.

27. Cheng SM, Chu KM and Lai JH: The modulatory mechanisms of fenofibrate on human primary T cells. Eur J Pharm Sci 40 316-324, 2010

28. Stienstra R, Mandard S, Tan NS, et al: The interleukin-1 receptor antagonist is a direct target gene of PPARalpha in liver. J Hepatol 46: 869-877, 2007.

29. Liu SF and Malik AB: NF-kappaB activation as a pathological mechanism of septic shock and inflammation. Am J Physiol Lung Cell Mol Physiol 290: L622-L645, 2006.

30. Delerive P, Gervois P, Fruchart JC and Staels B: Induction of IkappaB alpha expression as a mechanism contributing to the anti-inflammatory activities of peroxisome proliferator-activated receptor-alpha activators. J Biol Chem 275: 36703-36707, 2000.

31. Ji Y, Wang Z, Li Z and Liu J: Modulation of LPS-mediated inflammation by fenofibrate via the TRIF-dependent TLR4 signaling pathway in vascular smooth muscle cells. Cell Physiol Biochem 25: 631-640, 2010.

32. Galley HF: Oxidative stress and mitochondrial dysfunction in sepsis. Br J Anaesth 107: 57-64, 2011.
33. Maitra U, Chang S, Singh N and Li L: Molecular mechanism underlying the suppression of lipid oxidation during endotoxemia. Mol Immunol 47: 420-425, 2009.

34. Victor VM and De la Fuente M: Immune cells redox state from mice with endotoxin-induced oxidative stress. Involvement of NF-kappaB. Free Radic Res 37: 19-27, 2003.

35. Koksal GM, Sayilgan C, Aydin S, Oz H and Uzun H: Correlation of plasma and tissue oxidative stresses in intra-abdominal sepsis. J Surg Res 122: 180-183, 2004.

36. Olukman M, Sezer ED, Ulker S, Sözmen EY and Cınar GM: Fenofibrate treatment enhances antioxidant status and attenuates endothelial dysfunction in streptozotocin-induced diabetic rats. Exp Diabetes Res 2010: 828531, 2010

37. Boshra V and Moustafa AM: Effect of preischemic treatment with fenofibrate, a peroxisome proliferator-activated receptor- $\alpha$ ligand, on hepatic ischemia-reperfusion injury in rats. J Mol Histol 42: 113-122, 2011.

38. Lorente JA and Marshall JC: Neutralization of tumor necrosis factor in preclinical models of sepsis. Shock 24 (Suppl 1): $107-119,2005$.

39. Szakmany T, Hauser B and Radermacher P: N-acetylcysteine for sepsis and systemic inflammatory response in adults. Cochrane Database Syst Rev 9: CD006616, 2012.

40. Mermis JD and Simpson SQ: HMG-CoA reductase inhibitors for prevention and treatment of severe sepsis. Curr Infect Dis Rep 14: 484-492, 2012. 